\title{
Bayesian Pixel Density Estimation Modeling to Detect Human Sperm Sample Image Based on Sperm Head Shape
}

\author{
Candra Zonyfar ${ }^{1)^{*}}$, Kiki Ahmad Baihaqi ${ }^{2)}$ \\ 1)2) Universitas Buana Perjuangan Karawang, Indonesia \\ ${ }^{1)}$ candra@ubpkarawang.ac.id, ${ }^{2}$ kikiahmad@ubpkarawang.ac.id
}

Submitted : Sep 21, 2021 | Accepted : Oct 10, 2021 | Published : Oct 10, 2021

\begin{abstract}
Currently, there is a problem of the difficulty in classifying human sperm head sample images using different databases and measuring the accuracy of several different datasets. This study proposes a Bayesian Density Estimation-based model for detecting human sperm heads with four classification labels, namely, normal, tapered, pyriform, and small or amorphous. This model was applied to three kinds of datasets to detect the level of pixel density in images containing normal human sperm head samples. Experimental results and computational accuracy are also presented. As a method, this study labeled each human sperm head based on three shape descriptors using the formulas of Hu moment, Zernike moment, and Fourier descriptor. Each descriptor was also tested in the experiment. There was an increased accuracy that reached $90 \%$ after the model was applied to the three datasets. The Bayesian Density Estimation model could classify images containing human sperm head samples. The correct classification level was obtained when the human sperm head was detected by combining Bayesian $+\mathrm{Hu}$ moment with an accuracy rate of up to $90 \%$ which could detect normal human sperm heads. It is concluded that the proposed model can detect and classify images containing human sperm head objects. This model can increase accuracy, so it is very appropriate to be applied in the medical field.
\end{abstract}

Keywords: Human Sperm Heads (HSH); Classification; Hu moment; Zernike moment; Fourier descriptor.

\section{INTRODUCTION}

Image processing of human samples has grown widely from examining the skin to research on human sperm samples. Human sperm is unique in shape, which is characterized by the shape of the head, body, and tail. Healthy sperm has a standard head shape, called human sperm heads (HSH) (Iqbal et al., 2020). A method to detect images containing normal HSH based on certain criteria and image processing techniques is needed by medical laboratories (Yoon et al., 2008). The common problem for image processing experts to analyze HSH images is that each image contains sperm objects with different density levels (Di Caprio et al., 2015). This makes it difficult to detect healthy normal HSH and abnormal HSH (Sun et al., 2006). Therefore, a Bayesian model is proposed to identify the normal morphology of HSH.

HSH has a different morphology that requires a special method to measure the level of sperm density (Sun et al., 2006). Therefore, the concept of Bayesian Density Estimation (BDE) was used. Until now, little information explains how to detect normal HSH based on sperm morphology (Auger, 2010). Different results from different datasets also make it difficult to measure the standard of accuracy, hindering the normal and healthy assessment of HSH. The research questions in this article are (a) how to detect normal HSH from various HSH images? (b) how to find out the level of accuracy to identify normal HSH?

To answer these questions, a new model that combines Bayesian concepts with density estimation is proposed (Lu et al., 2013). The principle is to produce a method for detecting normal and healthy HSH using labeling and classification. The dataset used follows Ghasemian et al. (Ghasemian et al., 2015), Lammers, et al. (Lammers et al., 2014), and Aksoy et al. (Aksoy et al., 2012). The three types of datasets were then used in this study.

*Candra Zonyfar 
After the model was set up, several experiments were performed to identify and compare normal, tapered, pyriform, small, and amorphous HSH types (Riordon et al., 2019). This classification and labeling are useful for monitoring the quality of HSH. This study contributes to complement the shortcomings of previous methods to measure the accuracy of three kinds of datasets. In addition, from a computational point of view, no model can perform computations with satisfactory accuracy. To determine the usability of the proposed model, several tests were carried out to obtain the basic classification baseline.

To facilitate the implementation of this research, this paper is divided into several parts. In the second section, previous studies using Bayesian modeling techniques, classification, and labeling of $\mathrm{HSH}$, are reviewed. In the third part, this research presents HSH methods and features that can be processed from dataset images including the reasons for using the descriptors of Hu moment, Zernike moment, and Fourier descriptor. Each descriptor was also tested in the experiment. In the fourth section, the test results of the BDE model are presented after adding the three kinds of shape descriptors. The results of the accuracy value of the proposed model to detect normal HSH become the classification baseline. Summary and conclusions are then added to the fifth section.

\section{LITERATURE REVIEW}

\section{Point of Interest with Density Estimator}

The most visible part of a human sperm image sample is the head of the sperm (Figure 1). This sample is called HSH (Iqbal et al., 2020). A normal HSH image has the head as a point of interest position with a single best guess to detect the head position (Leung et al., 2011). The position of the sperm head is marked with $\theta^{*}$ and the point estimate position as $\hat{\theta}_{\text {or }} \hat{\theta}_{n}$. The letter $n$ in the point estimate $\hat{\theta}_{n}$ is the number of $\mathrm{n}$ observations or data points from a statistical experiment result for an estimate $\theta^{*}$. The number of points of interest positions is usually unknown, which will be combined with Hu moment, Zernike moment, and Fourier descriptor (Yadav et al., 2008). These three kinds of shape descriptors were used as classifiers $g^{*} \in \mathbb{G}_{\text {to }}$ fulfill the discrete regression equation $Y=g^{*}(X)+\ddot{I} \mu$ as an accuracy prediction formula so that an estimation equation was obtained, which $Y_{i}$ is affected $X_{i}$.

The data point of the HSH image indicated by $x \hat{\mathrm{a}} \%{ }^{\prime \prime}\left(x_{1}, x_{2}, \hat{\mathrm{a}} \biguplus_{1}, x_{n}\right)$ is an n-number vector or a series of points in $n$-dimensional real space, which means $x:=\left(x_{1}, x_{2}, \ldots, x_{n}\right) \in \mathbb{X}_{n}$. We thus emphasize that the dimension $n$ in the point estimator $\hat{\theta}_{n_{\text {of }}} \theta^{*} \in \mathbb{T}$ will result in:

$$
\hat{\theta}_{n}:=\hat{\theta}_{n}\left(z:=\left(x_{1}, x_{2}, \ldots, x_{n}\right)\right)=\hat{\theta}_{n}: \mathbb{X}_{n} \rightarrow \mathbb{T}
$$

Thus, the normal HSH shape can be represented by $\mathbb{T}$ that has one-point estimate $\theta^{*} \in \mathbb{T}$ provided that the normal HSH has the pattern $x \hat{a}^{\wedge} \mathbb{X}_{n}$ as an independent part of the real number $\mathbb{R}^{n}$ and is randomly distributed following a general vector pattern $X=\left(X_{1}, X_{2}, \hat{\mathrm{a}} €_{\mid}^{\prime}, X_{n}\right)$ until $X_{1}, X 2, \hat{\mathrm{a}} €_{\mid}^{\prime} X_{n}$.

\section{Hu Moment, Zernike moment, Fourier descriptor}

Hu moments was used to obtain pixels collected into an HSH object (Chang et al., 2017). Each object was then assigned to a set of morphological shape classes. The Zernike moment was used to divide the segment of pixels from the image containing points of interest (thin pixels) that are not perfectly shaped or have real vectors of discrete values so that they are grouped to form a discrete function $\left\{\left(x_{1}, y_{2}\right), \hat{\mathrm{a}} €^{1},\left(x_{m}, y_{m}\right)\right\}$ to be projected into a normalized image form (Flusser et al., 2016).

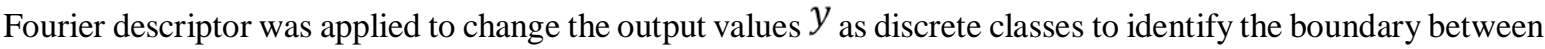
the object and the background color. With the form of a discrete set, the function $f(x)$ can produce a powerful classifier. After all moments are formed, Bayesian principles are added to strengthen the model's performance in classifying all HSH images. 


\section{Bayesian Density Estimation}

Previous studies demonstrated the need to have computational modeling to identify normal HSH using different approaches (Chang et al., 2017). However, finding a cost-efficient and widely available approach is a complicated and expensive process.

BDE provides opportunities for further development. The Bayesian theorem has the basic form of conditional probability as the basis for predicting the location of sperm in an image:

$$
p\left(M_{A} \mid F\right)=\frac{P\left(M_{A} \cap F\right)}{P(F)}
$$

Where $p\left(M_{A} \mid F\right)$ is the posterior probability that arises from the image of the sample set $\mathrm{A}$ and $\mathrm{F}$ is the feature of the object being searched for. BDE was used to observe images that contained sperm head objects but did not have standard parameters.

Because the position of the sperm head tended to be uncertain in an image, an uncertainty parameter with a random value was added (Gómez \& Maddison, 2020). The set of random values formed subset $D$ that represents the data found and the model parameter $\theta$, so for each inference, the combined probability distribution $P(D, \hat{I} s)$ of all the random sums of the two parameters was obtained. Thus, the combined distribution is written as:

$$
P(D, \hat{\mathrm{I}})=P(\hat{\mathrm{I}}) P(D \mid \hat{\mathrm{I}} s)
$$

\section{Source dataset with HSH images}

Several recent datasets have been proposed by Ghasemian, et al. (2015) using normal HSH detection techniques. The dataset contains 1,457 576×764 image samples successfully used to detect normal HSH. The parameters used are shape parameters (morphology), not sperm classification. Another researcher, Lammers (Lammers et al., 2014), proposed a dataset containing 250 image samples applied to detect normal HSH. The third dataset used was proposed by Aksoy et al. (Aksoy et al., 2012) with 67 image samples. All datasets were then mixed and grouped into three subsets, namely dataset1, dataset2, and dataset3. Each dataset contains images of various sizes to be grouped into five morphological classes, namely normal, tapered, pyriform, small, and amorphous.

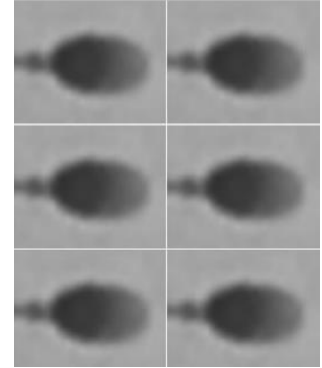

(a)

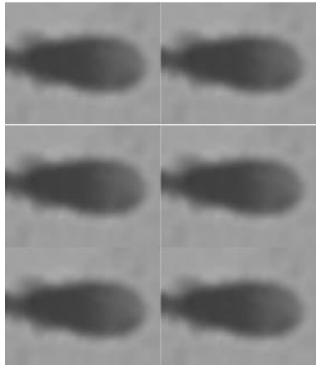

(b)

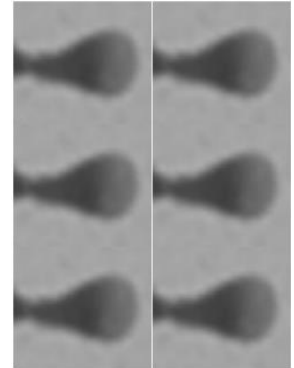

(c)

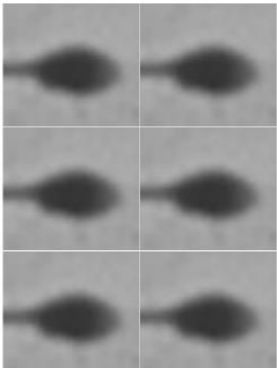

(d)

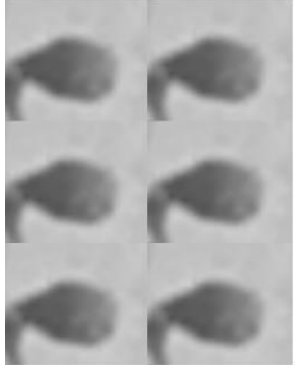

(e)

Fig. 1 is an example of an HSH shape based on normal (a), tapered (b), pyriform (c), small (d), and amorphous (e) morphology shapes.

For this study, a model to represent and identify normal HSH and differentiate it from abnormal HSH based on the morphology above was constructed. 


\section{METHOD}

The classification in Section 2 above was used for each test based on a comparison of three datasets distributed to five classes.

The dataset was grouped into three subsets, namely dataset 1 , dataset 2 , and dataset 3 , which represented the objectives of training ( $60 \%$ of the entire dataset), validating (20\%), and testing $(20 \%)$. Table 4 shows the size and distribution of the classes on each partition. The experimental steps are shown in detail in Figure 2.

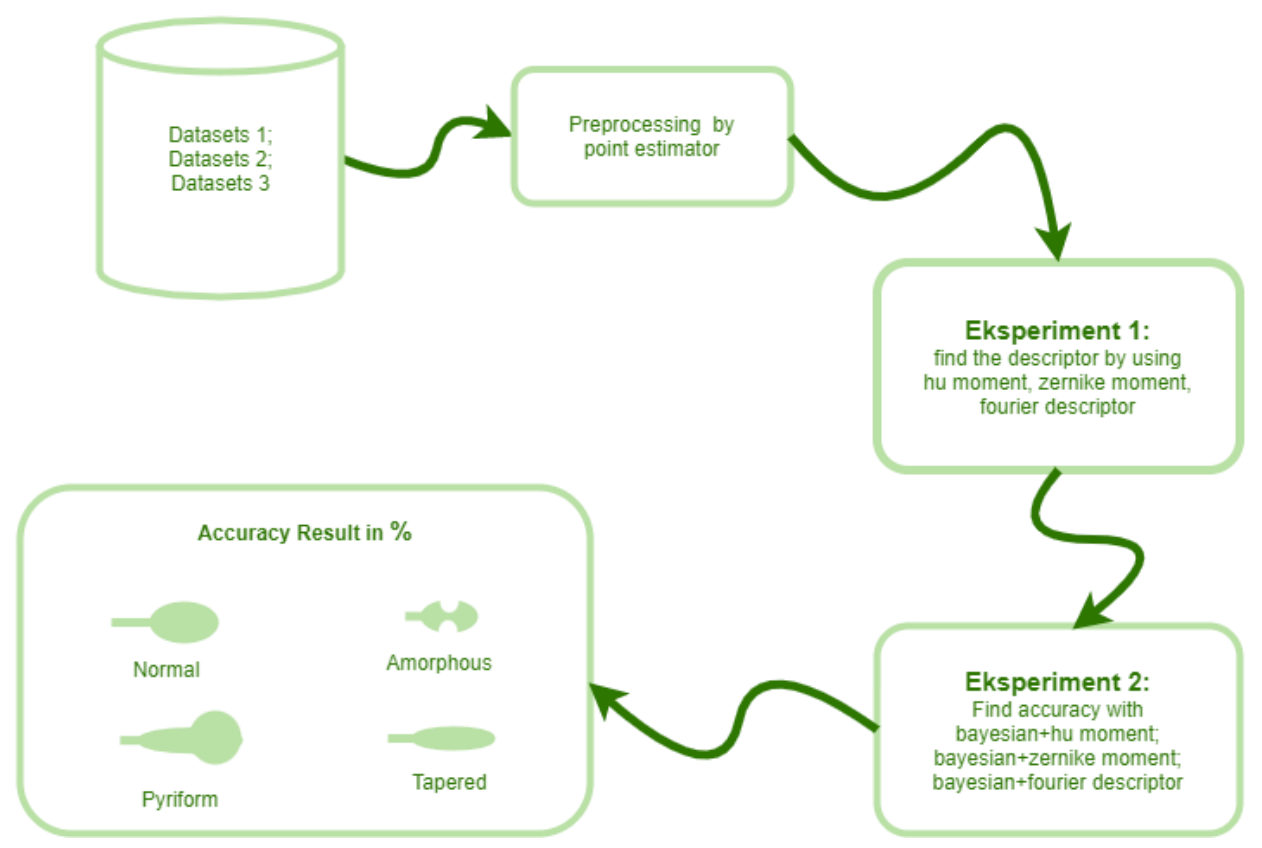

Fig. 2. Flowchart of HSH detection based on the morphology of the HSH shape using BDE

\section{ANALYSIS AND DISCUSSION}

In this study, BDE modeling was made to detect normal HSH by adding shape morphology parameters. The parameters used to detect the HSH shape are Hu moment, Zernike Moment, and Fourier descriptor (Yadav et al., 2008). These parameters were used to classify the HSH of the dataset into five classes, namely normal, tapered, pyriform, small, and amorphous (Figure 3). The accuracy obtained from the three parameters was then compared with the accuracy using BDE (Figures 3-7) (Shaker et al., 2017).

Figure 3 shows the original image dataset sample after adding a shape morphology descriptor compared with the images after processing with BDE ( $\mathrm{Lu}$ et al., 2013). The original image and the processed image with the descriptor still have a fairly high noise compared to the image processing by BDE (Shen et al., 2013). BDE can process images so that they have sharper edges and more visible pixel-dividing lines. 


\begin{tabular}{|c|c|c|c|c|c|}
\hline & \multirow{2}{*}{ Normal shape } & \multicolumn{4}{|c|}{ Abnormal shape } \\
\hline & & Tapered & Pyriform & Small & Amorphous \\
\hline \multicolumn{6}{|l|}{ Original image } \\
\hline \multicolumn{6}{|l|}{$\begin{array}{l}\text { After applying the } \\
\text { shape morphology } \\
\text { descriptor }\end{array}$} \\
\hline After applying & & & & & \\
\hline Bayesian density & & & & & \\
\hline & & & & & \\
\hline
\end{tabular}

Figure 3. Comparison of image processing results: original, after descriptors, after BDE

\section{Classification of normal HSH using density estimator}

The dataset came from the Internet from three sources combined into one and then preprocessed to separate unused images. All images were then randomized. Then, modeling was made with five shape descriptors that divided the HSH samples based on the labeling of the Hu moment descriptor, Zernike moment descriptor, and Fourier Descriptor (YE Bin, 2003). The labeling was based on shape and morphology. There are five shapes, namely, normal, tapered, pyriform, and small or amorphous.

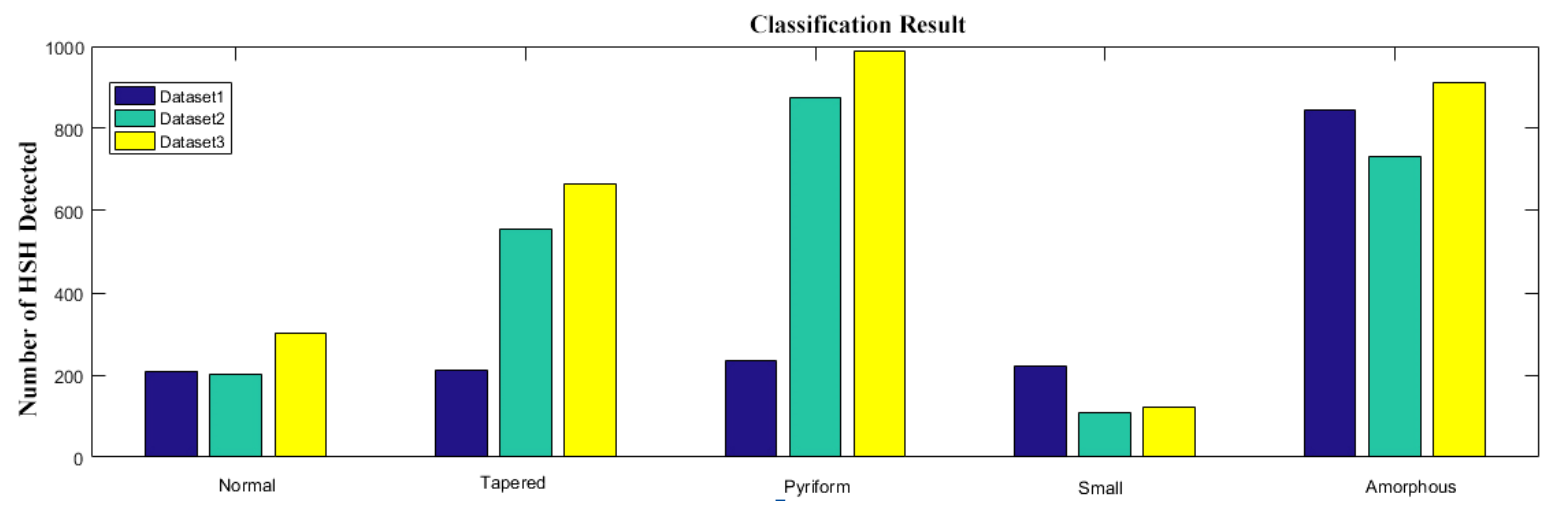

Fig. 4 Normal HSH classification results using density estimator

Figure 4 shows the results of normal HSH classification using only a density estimator. Each bar chart displays the results of labeling on images with normal HSH. The detection results show that there are 200-300 images of normal HSH from dataset1. Then, the density estimator can predict the highest in pyriform HSH and amorphous HSH which is close to 1,000 images. This means that the density estimator can predict abnormal HSH (i.e., pyriform and amorphous) but not normal HSH. 


\section{Detected image using the shape morphology descriptor}

There are three kinds of shape morphological descriptors or shape moment descriptors, namely Hu moment, Zernike moment, and Fourier descriptors (Rashid et al., 2010). Three datasets were retested using these three descriptors to find out the number of detected images. Figure 5 shows that the Hu moment can only detect normal HSH while the other four shapes have low accuracy. Zernike moment can also do the same thing, followed by the Fourier descriptor.

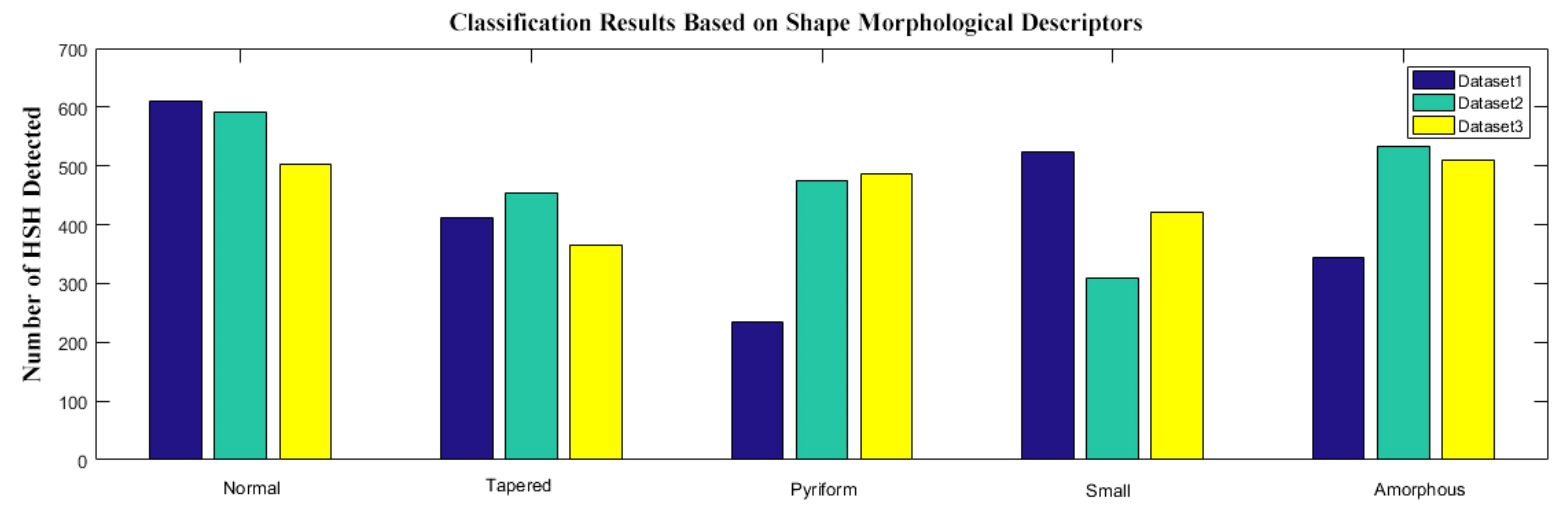

Fig. 5 Experimental results with shape morphology descriptors (Hu moment, Zernike moment, Fourier descriptor)

It appears that for normal HSH detection. Hu moment can be used. It can also be used to detect small HSH. To detect normal and amorphous HSH, Zernike moment can be used. In the Fourier descriptor, there is only moderate accuracy. Overall, Hu moment can outperform Zernike moment and Fourier descriptor (Rashid et al., 2010). The test results indicate a high accuracy performance in normal HSH samples but not in other classes. From this condition, we developed BDE to improve accuracy in the normal HSH class based on the above classification.

\section{Detected image using BDE}

BDE is the model proposed in this study (Shen et al., 2013). Three datasets were tested using BDE which resulted in the highest accuracy in the normal HSH class $(\alpha=0.05)$ while the accuracy for other classes tended to continue to decline (Figure 6).

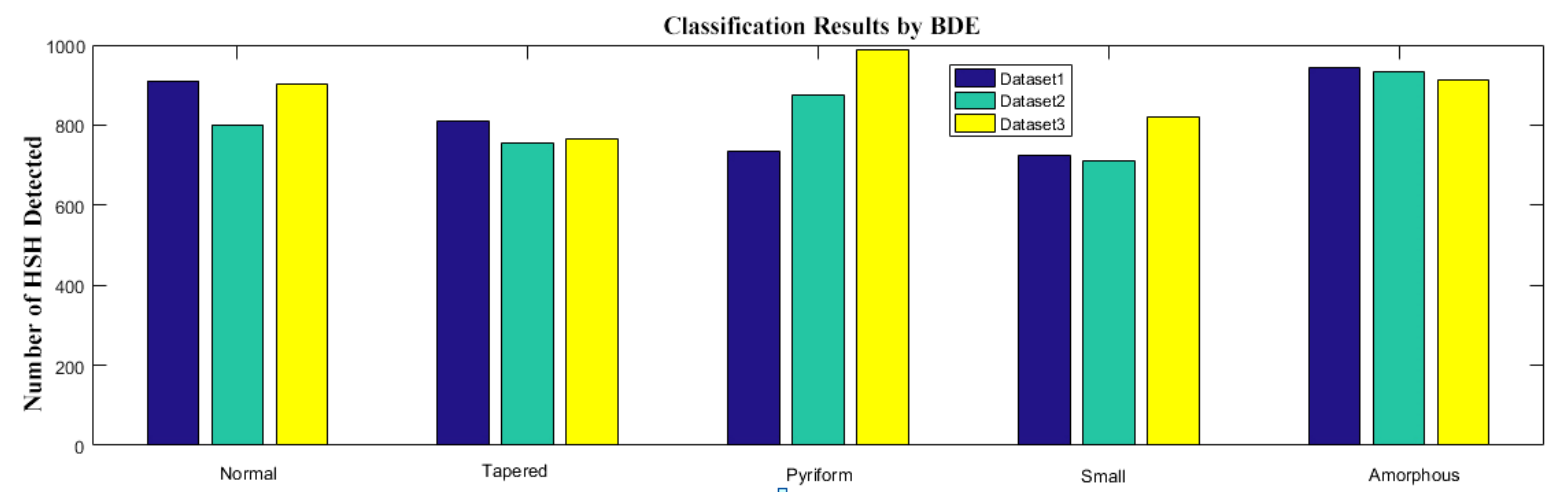

Fig. 6 Detected image using BDE

The test results show the existence of high performance and accuracy in the Bayes model proposed here. There are 900 correctly detected images (Figure 6). The proposed Bayes model can classify HSH samples in all shape morphology classes reaching the highest level compared to other models (Figure 6). The number of images detected with the correct classification reached the highest obtained when the image contained HSH detected by Bayesian $+\mathrm{Hu}$ moment. However, when BDE is used to detect images containing tapered $\mathrm{HSH}$, there is a decrease in performance. The same thing happened to HSH with other morphology shapes, namely, pyriform, small, and 
amorphous shapes. This means that there are weaknesses in the proposed BDE model when it comes to detecting different shape classes.

\section{Percentage of classification accuracy using BDE}

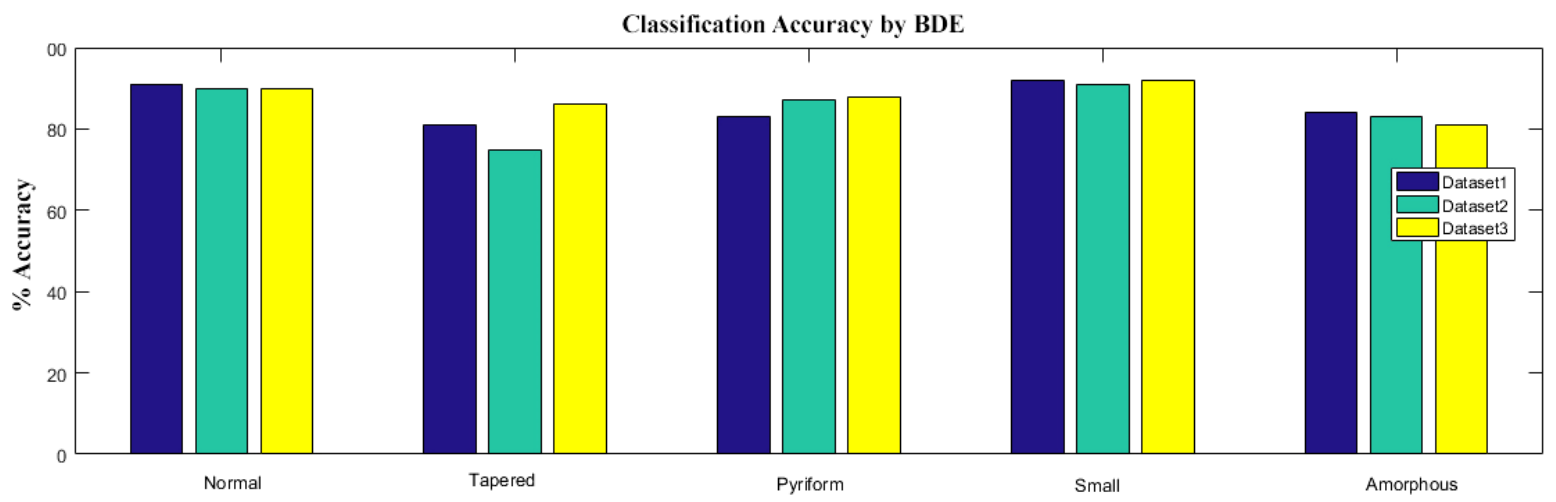

Fig. 7 Classification efficiency of BDE model

To improve the accuracy of prediction and classification, BDE which contained a combination of shape morphological descriptors and Bayesian principle was applied. Figure 7 shows that after adding BDE, there is an increase in accuracy for dataset1 which can detect normal HSH up to $90 \%$. Then, the percentage of accuracy on tapered HSH is obtained in dataset 2 followed by small and amorphous HSH. These results show that the shapes with the highest accuracy are normal and tapered while other shapes have lower accuracy even though a combination of shape morphology descriptors and BDE has been applied.

\section{CONCLUSION}

This study aims to find Bayesian modeling has been achieved. The proposed model is based on BDE which can work well for three kinds of datasets containing normal HSH. Experimental results show that our approach can successfully identify normal HSH. The proposed model is even able to detect higher HSH than other models. The BDE model, according to the experimental results, provides several images successfully detected with a high level of accuracy.

This classification model can be used to support the medical community in obtaining healthy HSH and applied to assisted reproduction technology. The proposed model can detect normal HSH using labeling and classification based on shape morphology, namely, normal, tapered, pyriform, small, and amorphous HSH types. This classification and labeling can help monitor normal HSH quality. It is suggested that future research add other descriptors using more sophisticated techniques such as observing texture, depth image, or grayscale segmentation.

\section{ACKNOWLEDGMENT}

The authors would like to acknowledge a research grant from Ministry of Education, Culture, Research, and Technology Republic of Indonesia (Kemendikbud Ristek) for the funding support of the research project (Hibah Penelitian Dosen Pemula 2021 No:1867/E4/AK.04/2021).

\section{REFERENCES}

Aksoy, E., Aktan, T. M., Duman, S., \& Cuce, G. (2012). Assessment of Spermatozoa Morphology under Light Microscopy with Different Histologic Stains and Comparison of Morphometric Measurements. International Journal of Morphology, 30(4), 1544-1550. https://doi.org/10.4067/s071795022012000400045

Auger, J. (2010). Assessing human sperm morphology: Top models, underdogs or biometrics? Asian Journal of *Candra Zonyfar 
Andrology, 12(1), 36-46. https://doi.org/10.1038/aja.2009.8

Chang, V., Heutte, L., Petitjean, C., Härtel, S., \& Hitschfeld, N. (2017). Automatic classification of human sperm head morphology. Computers in Biology and Medicine, 84, 205-216. https://doi.org/10.1016/j.compbiomed.2017.03.029

Di Caprio, G., Ferrara, M. A., Miccio, L., Merola, F., Memmolo, P., Ferraro, P., \& Coppola, G. (2015). Holographic imaging of unlabelled sperm cells for semen analysis: A review. Journal of Biophotonics, 8(10), 779-789. https://doi.org/10.1002/jbio.201400093

Flusser, J., Suk, T., \& Zitová, B. (2016). 2D and 3D Image Analysis by Moments. 2D and 3D Image Analysis by Moments, 1-529. https://doi.org/10.1002/9781119039402

Ghasemian, F., Mirroshandel, S. A., Monji-Azad, S., Azarnia, M., \& Zahiri, Z. (2015). An efficient method for automatic morphological abnormality detection from human sperm images. Computer Methods and Programs in Biomedicine, 122(3), 409-420. https://doi.org/10.1016/j.cmpb.2015.08.013

Gómez, R. A., \& Maddison, D. R. (2020). Novelty and emergent patterns in sperm: Morphological diversity and evolution of spermatozoa and sperm conjugation in ground beetles (Coleoptera: Carabidae). Journal of Morphology, 281(8), 862-892. https://doi.org/10.1002/jmor.21144

Iqbal, I., Mustafa, G., \& Ma, J. (2020). Deep learning-based morphological classification of human sperm heads. Diagnostics, 10(5). https://doi.org/10.3390/diagnostics10050325

Lammers, J., Splingart, C., Barrière, P., Jean, M., \& Fréour, T. (2014). Double-blind prospective study comparing two automated sperm analyzers versus manual semen assessment. Journal of Assisted Reproduction and Genetics, 31(1), 35-43. https://doi.org/10.1007/s10815-013-0139-2

Leung, C., Lu, Z., Esfandiari, N., Casper, R. F., \& Sun, Y. (2011). Automated sperm immobilization for intracytoplasmic sperm injection. IEEE Transactions on Biomedical Engineering, 58(4), 935-942. https://doi.org/10.1109/TBME.2010.2098875

Lu, L., Jiang, H., \& Wong, W. H. (2013). Multivariate density estimation by Bayesian sequential partitioning. Journal of the American Statistical Association, 108(504), 1402-1410. https://doi.org/10.1080/01621459.2013.813389

Rashid, O., Al-Hamadi, A., \& Michaelis, B. (2010). Utilizing invariant descriptors for finger spelling American sign language using SVM. Lecture Notes in Computer Science (Including Subseries Lecture Notes in Artificial Intelligence and Lecture Notes in Bioinformatics), 6453 LNCS(PART 1), 253-263. https://doi.org/10.1007/978-3-642-17289-2_25

Riordon, J., McCallum, C., \& Sinton, D. (2019). Deep learning for the classification of human sperm. Computers in Biology and Medicine, 111(June). https://doi.org/10.1016/j.compbiomed.2019.103342

Shaker, F., Monadjemi, S. A., Alirezaie, J., \& Naghsh-Nilchi, A. R. (2017). A dictionary learning approach for human sperm heads classification. Computers in Biology and Medicine, 91, 181-190. https://doi.org/10.1016/j.compbiomed.2017.10.009

Shen, W., Tokdar, S. T., \& Ghosal, S. (2013). Adaptive Bayesian multivariate density estimation with Dirichlet mixtures. Biometrika, 100(3), 623-640. https://doi.org/10.1093/biomet/ast015

Sun, F., Ko, E., \& Martin, R. H. (2006). Is there a relationship between sperm chromosome abnormalities and sperm morphology? Reproductive Biology and Endocrinology, 4(February). https://doi.org/10.1186/14777827-4-1

Yadav, R. B., Nishchal, N. K., Gupta, A. K., \& Rastogi, V. K. (2008). Retrieval and classification of objects using generic Fourier, Legendre moment, and wavelet Zernike moment descriptors and recognition using joint transform correlator. Optics and Laser Technology, 40(3), 517-527. https://doi.org/10.1016/j.optlastec.2007.08.007

YE Bin, P. J. (2003). Improvement and invariance analysis of pseudo-Zernike moments. Journal of Image and Graphics, 3.

Yoon, S. Y., Jellerette, T., Salicioni, A. M., Hoi, C. L., Yoo, M. S., Coward, K., Parrington, J., Grow, D., Cibelli, J. B., Visconti, P. E., Mager, J., \& Fissore, R. A. (2008). Human sperm devoid of PLC, zeta 1 fail to induce

*Candra Zonyfar 
$\mathrm{Ca} 2+$ release and are unable to initiate the first step of embryo development. Journal of Clinical Investigation, 118(11), 3671-3681. https://doi.org/10.1172/JCI36942 